\title{
Low latency and energy efficient cluster based routing design for wireless sensor network
}

\author{
Basavaraj G.N' ${ }^{1}$, Jaidhar C.D ${ }^{2}$ \\ ${ }^{1}$ Department of Computer Science and Engineering, Jawaharlal Nehru Technological University, Anantpur, India \\ ${ }^{2}$ Department of Information Technology, National Institute of Technology, Karnataka, India
}

\begin{tabular}{l} 
Article Info \\
\hline Article history: \\
Received Jun 22, 2018 \\
Revised Aug 29, 2018 \\
Accepted Sep 14, 2018 \\
\hline
\end{tabular}

\section{Keywords:}

Clustering

Cross layer

Hop transmission

Lifetime

WSN

\begin{abstract}
Wireless sensor network (WSN) has attained wide adoption across various sectors and is considered to be key component of future real-time application such as BigData, Internet of things (IoT) etc. The modern application requires low latency and scalable real-time data access considering heterogeneous network. However, provisioning low latency real-time data access incurs energy overhead among sensor device. Clustering technique aided in providing scalability and minimizing energy consumption among sensor device. However, it incurs energy overhead among cluster head and sensor device closer to sink. To address, many optimization technique is been presented in recent time for optimal cluster selection. However, these technique are designed considering homogenous network. To address, this work presented Low Latency and Energy Efficient Routing (LLEER) design for heterogeneous WSN. The LLEER adopts multi-objective function such as connectivity, connection time, radio signal strength, coverage time, and network traffic for cluster head and hop node selection. Experiment are conducted to evaluate LLEER design shows significant performance improvement over state-of-art model in terms of network lifetime considering total node death, first node death, and loss of connectivity, communication overhead, and packet transmission latency. Proposed LLEER brings a good trade-off between energy efficiency, and latency requirement of future realtime application.
\end{abstract}

Copyright $\odot 2019$ Institute of Advanced Engineering and Science. All rights reserved.

\section{Corresponding Author:}

Basavaraj G.N,

Department of Computer Science and Engineering,

Jawaharlal Nehru Technological University,

Anantpur, Andhra Pradesh, India.

Email: basavarajgn@gmail.com

\section{INTRODUCTION}

With the growth of sensor technologies, wireless sensor network (WSN) has attained as a most sorted out network architecture of future wireless based application and communication services. For example, it is adopted in the field of health care monitoring, intelligent transportation, robotics, industrial manufacture, etc [1]. Further, WSN has been applied in various real-time and non-real-time smart and intelligent applications, such as, Training Monitoring System [2], smart city [3] and other application[4], etc. A foremost responsibility of the WSNs is accurately sensing and gathering useful information, such as, the measurements of humidity, air quality, chemical information, and biomedical and yielding sensed big data for future analysis [5]. At the same instance, cloud-computing enabled technologies, such as Fog-RAN [6] and Cloud-RAN [7], offer WSNs with the benefits of computation, communication and storage resources [8], as well as a promising method to manage and process of massive aggregated data [9].

The sensor device are placed in hazardous location were recharging or replacing of battery is impossible, where human monitoring comprises huge risk. The sensor device can be event or time driven, in 
both cases the energy of battery is depleted exponentially. The sensed data are either transmitted to the neighbouring devices or to the base station. In some scenario same data is transmitted to base station. Thus affecting energy efficiency of WSN. To overcome the redundancy issue and make network more energy efficient data aggregation technique is used in [10]. To provision real-time access [11] to sensor data for reliable industries application, accurate gathering and processing of information is required [12]. However, performing data aggregation possess a challenge for which a solution is presented in [13]. To provide efficient data aggregation, energy preservation scheme is presented [14]. Energy preservation routing design adopting clustering protocol such as HEED and LEACH has been presented in [15]. However, they incurs energy overhead among cluster head and are not efficient for relatively large network [16] because direct transmission of data through cluster head toward sink is not a feasible strategy for such network. To overcome [17] designed a routing technique for hop device selection for cluster network to enhance lifetime of WSNs. However, it incurs high communication overhead due to channel contention among hop device and cluster device and optimizing it is NP-deterministic.

In [18] presented an energy efficient design for large sensor network adopting fuzzy based clustering approach. However, lifetime performance is not efficient, the cluster head devices closer to the base station dies rapidly. To overcome the issues, [19] presented a clustering design using type-2 fuzzy logic (T2FL). The model distributed load among sensor devices which aided in improving the lifetime of sensor network. However, T2FL clustering approach is designed considering homogenous network. Therefore, the future routing model should need to consider the heterogeneity requirement of wireless sensor network and its applications [20-23]. To accumulate and communicate these data in real-time efficient design are required. In [24] presented a data gathering model, [25] presented a efficient data routing model adopting clustering approach, [26] presented data forecasting technique for cluster based WSNs, and [27], [28] presented cross layer approach for cluster based WSNs. The model [24-28] minimized energy consumption of sensor device. However, did not considered and failed to minimize data access latency. To address latency issues, optimization function adopting evolutionary computing for cluster formation is presented [29]. However, extensive analysis carried out in [30] shows that evolutionary computing for heterogeneous wireless sensor network incurs computation overhead among sensor nodes. Thus, affecting the performance of WSN. In [31] presented clustering approach for heterogeneous WSN using tree structure. The model considered link quality and packet loss rate to minimize energy consumption of sensor node. However, the model did not considered data access latency.

To overcome the research challenges this work presented low latency and energy efficient routing (LLEER) design for WSN considering heterogeneous and mobile sensor network. The LLEER technique adopt both cluster and hop based transmission. Firstly, this work present an efficient cluster head selection technique using multi objective function such as connectivity, connection time, radio signal strength and coverage time. The cluster head aggregates data from its cluster member. Secondly, LLEER techniques build a routing/transmission path formation to collect and aggregates data information from cluster head toward sink. This transmission is carried out directly or through set of transmission path nodes. The transmission path nodes is built using multi-objective function similar to cluster head selection along with traffic parameter. Data aggregation is performed both in cluster head and as well as in transmission path node thus minimizing traffic and improving energy efficiency of sensor network.

The Contribution of research work is as follows:

a. This paper presented a low latency and energy efficient routing design for mobile and heterogeneous WSN.

b. Our model adopts both cluster and hop based transmission.

c. Data aggregation is performed both at cluster head level and Transmission path node level thus minimizing network load and improving energy efficiency of sensor network.

d. No prior work has considered cluster head and hop selection design considering heterogeneous and mobile sensor environment.

e. No prior work has considered lifetime performance evaluation considering total node death, first node death and loss of connectivity considering heterogeneous WSNs.

f. Performance of lifetime analysis is carried out considering total device death, first sensor device death, and loss of connectivity.

g. The model minimize energy consumption of sensor device, minimize latency and communication overhead of real-time data access and maximize lifetime of WSN.

The paper organization is as follows: The proposed low latency and energy efficient clustering technique are presented in Section II. The simulation results and the experimental study are presented in section III. Result and discussion is presented in penultimate section. The concluding remark and future work is discussed in the last section. 


\section{LOW LATENCY AND ENERGY EFFICIENT ROUTING DESIGN FOR WSN}

Here the author proposes a low latency and energy efficient routing (LLEER) design for wireless sensor network. The LLEER model is composed of initialization stage and transmission stage. In initialization stage, cluster formation and transmission path formation (TPF) is carried out to obtain ideal path among cluster member $(\mathrm{CM})$ and sink/base station i.e., represented as intra cluster and transition path formation communication. Then, the transmission stage is initialized to transmit packets from the cluster member nodes to the sink. The architecture of proposed LLEER is presented in below Figure 1.

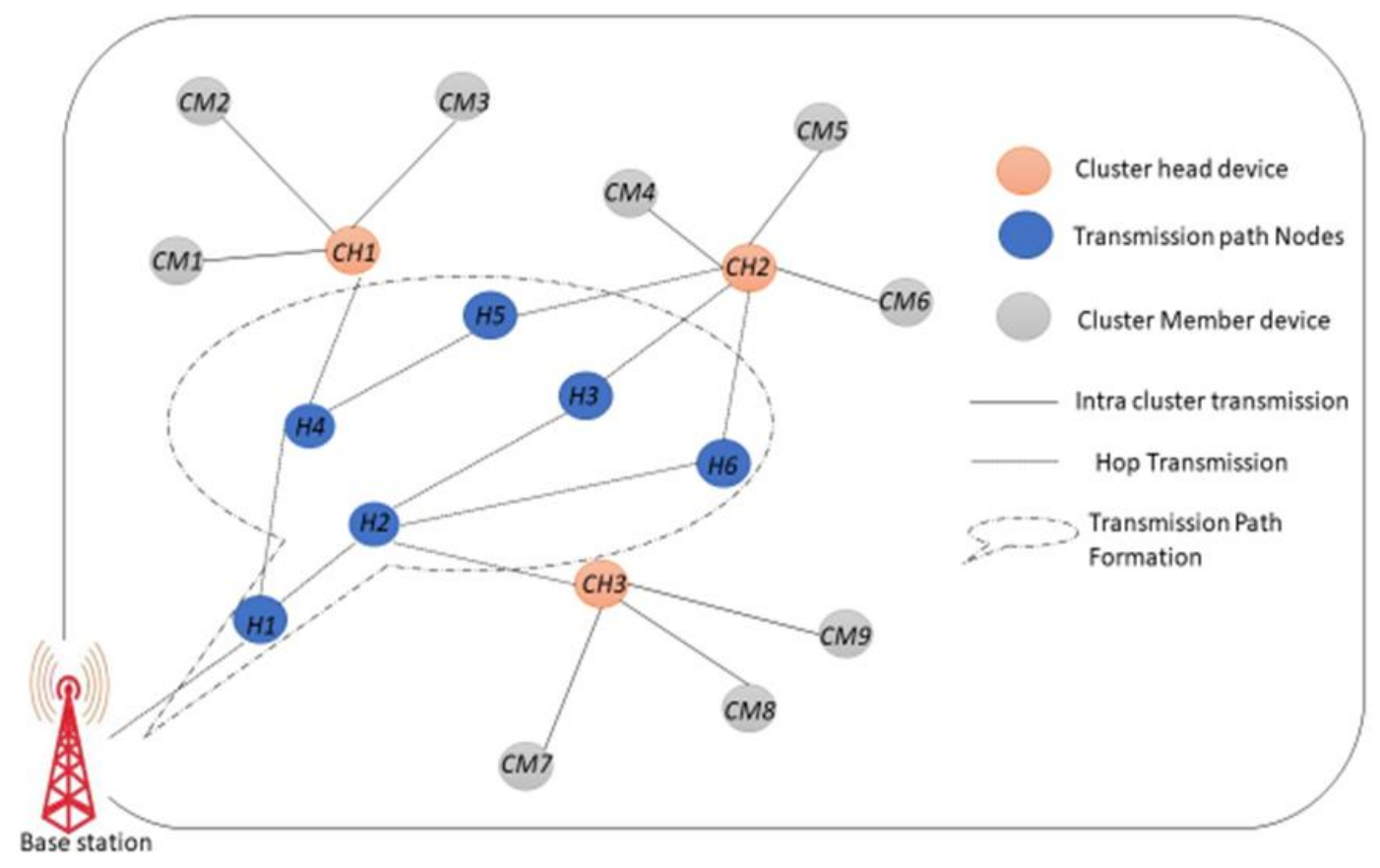

Figure 1. Architecture of Proposed LLEER Model

\subsection{Initialization stage}

Initialization stage is composed of intra cluster and transmission path formation communication process. In intra cluster communication process, all the sensor device elects the cluster head $(\mathrm{CH})$ using threshold parameter, and builds cluster with best radio signal strength (RSS), connectivity, connection time and coverage time. Post completion of intra cluster communication, transmission path formation communication is initialized, which obtains packets from its $\mathrm{CH}$ and then transmit the aggregated data towards its sink. Let consider all the $\mathrm{CH}$ to be associated with transmission path node (TPN), and all the TPN associated with sink which builds the TPF.

\subsection{Intra cluster communication phase}

Let consider a large and densely populated wireless sensor network. In initialization stage, the sensor device location is identified using beacon signal. Once the sensor devices location are identified, $\mathrm{CH}$ selection methodology is used to select the $\mathrm{CH}$. In this work, the $\mathrm{CH}$ selection is based on threshold parameter $V_{\mathbb{N C}}^{n}$, connectivity $H_{\mathbb{N} C}^{n}$, connection time $\delta E_{\mathbb{N} \mathbb{C}}^{\mathbb{C}}(u, u+t)$, and coverage time $\delta J_{\mathbb{N} \mathbb{C}}^{\mathbb{C}}(u, u+t)$. Post completion of CH selection, TPF construction is initialized. In this work the threshold parameter $V_{\mathbb{N C}}^{n}$ is computed using (1) by adding flag parameter with multiplication of factors such as remaining energy, number of neighbouring sensor devices, current speed and coverage distance of sensor devices. Let $G_{C}$ be the flag (set, $G_{C}=0$ for senor devices having high probability to act as $\mathrm{CH}$ for present round based on $V_{\mathbb{N C}}^{n}$ and $G_{C}=1$ for preceding round $\mathrm{CH}), F_{\mathbb{C}}^{c}$ is the present sensor devices energy, $O_{\mathbb{C}}^{c}$ is the number of cluster member nodes on this round, $W_{\mathbb{C}}^{c}$ is the sensor device present speed, $S_{\mathbb{C}}^{c}$ is the present coverage radius of sensor device, $F_{\mathbb{N}}^{\mathbb{M}}$ is the initial energy of senor device, $O_{\mathbb{N}}^{\mathbb{M}}$ is the maximum number of cluster members per round, $S_{\mathbb{N}}^{\mathbb{M}}$ is the sensor device maximum coverage radius, and $W_{\mathbb{N}}^{\mathbb{M}}$ is the sensor devices maximum speed.

Here we consider $\left(\left(W_{\mathbb{N}}^{\mathbb{M}}-W_{\mathbb{C}}^{c}\right) /\left(W_{\mathbb{N}}^{\mathbb{M}}+W_{\mathbb{C}}^{c}\right)\right)$ instead of $\left(W_{\mathbb{N}}^{\mathbb{M}} / W_{\mathbb{C}}^{c}\right)$ in order to avoid selection of high mobility sensor device as $\mathrm{CH}$. Then, the $\left(\left(S_{\mathbb{N}}^{\mathbb{M}}-S_{\mathbb{C}}^{c}\right) /\left(S_{\mathbb{N}}^{\mathbb{M}}+S_{\mathbb{C}}^{c}\right)\right)$ is considered to select $\mathrm{CH}$ with

Low latency and energy efficient cluster based routing design for wireless sensor network (Basavaraj G.N) 
maximum coverage distance. Subsequently, the $O_{\mathbb{N}}^{\mathbb{M}}$ is the derivative from current number of sensor devices $N_{c}$ or expected number of sensor devices in each cluster, which is obtained in (10). The sensor devices with maximum number of cluster member, radio signal strength, remaining energy and connection time can be selected as $\mathrm{CH}$.

$$
V_{\mathbb{N} \mathbb{C}}^{n}=G_{C}+\left(\frac{o_{\mathbb{C}}^{c}}{o_{\mathbb{N}}^{\mathbb{M}}-O_{\mathbb{C}}^{c}} * \frac{F_{\mathbb{N}}^{\mathbb{M}}-F_{\mathbb{C}}^{c}}{F_{\mathbb{N}}^{\mathbb{M}}} * \frac{W_{\mathbb{N}}^{\mathbb{M}}-W_{\mathbb{C}}^{c}}{W_{\mathbb{N}}^{\mathbb{M}}+W_{\mathbb{C}}^{c}} * \frac{S_{\mathbb{N}}^{\mathbb{M}}-S_{\mathbb{C}}^{c}}{S_{\mathbb{N}}^{\mathbb{M}}+S_{\mathbb{C}}^{c}}\right)
$$

It is considered that two dimensional network position of $\mathrm{CH} \mathbb{C}$ and sensor device $\mathbb{N}$ at instance $u$ is described as follows

$$
\begin{array}{ll}
A_{\mathbb{C}}=a_{\mathbb{C}}+w_{\mathbb{C}} * \cos \theta_{\mathbb{C}} u ; & B_{\mathbb{C}}=b_{\mathbb{C}}+w_{\mathbb{C}} * \sin \theta_{\mathbb{C}} u \\
A_{\mathbb{N}}=a_{\mathbb{N}}+w_{\mathbb{N}} * \cos \theta_{\mathbb{N}} u ; & B_{\mathbb{N}}=b_{\mathbb{N}}+w_{\mathbb{N}} * \sin \theta_{\mathbb{N}} u,
\end{array}
$$

where $(a, b)$ is the location of primary sensor device, $\theta$ is the angle of moving path among $(a, b), w$ is the speed, $u$ is the connection time and $A_{\mathbb{C}}$ or $A_{\mathbb{N}}$ is the present location of $\mathbb{C}$ or $\mathbb{N}$ at instance $u$. Then, the subscript $(\mathbb{N}, \mathbb{C})$ corresponds to sensor device $\mathbb{N}$ and $\mathrm{CH} \mathbb{C}$ respectively. Let the $E_{\mathbb{N} \mathbb{C}}^{\mathbb{C}}(u)$ be the distance among $\mathrm{CH}$ and cluster member at any instance $u$ is obtained as follows

$$
\left[E_{\mathbb{N} \mathbb{C}}^{\mathbb{C}}(u)\right]^{2} \geq\left[\left(A_{\mathbb{C}}-A_{\mathbb{N}}\right)^{2}+\left(B_{\mathbb{C}}-B_{\mathbb{N}}\right)^{2}\right]
$$

Each sensor device obtains advertisement message at instance $u=0$ from any one of the CHs. Therefore, (3) can be simplified as follows

$$
\left[E_{\mathbb{N} \mathbb{C}}^{\mathbb{C}}(u)\right]^{2} \geq\left[\left(A_{\mathbb{C}}-A_{\mathbb{N}}\right)^{2}+\left(B_{\mathbb{C}}-B_{\mathbb{N}}\right)^{2}\right] \text {, if } u=0
$$

Now, the connection instance $\delta E_{\mathbb{N} \mathbb{C}}^{\mathbb{C}}(u+u+t)$ is the difference among $E_{\mathbb{N} \mathbb{C}}^{\mathbb{C}}(u)$ and $\delta E_{\mathbb{N} \mathbb{C}}^{\mathbb{C}}(u+t)$ at instance $u$ and $u+t$. Let $\delta E_{\mathbb{N} \mathbb{C}}^{\mathbb{C}}(u+u+t)$ can be obtained from (4).

$$
\delta E_{\mathbb{N} \mathbb{C}}^{\mathbb{C}}(u+u+t)=E_{\mathbb{N} \mathbb{C}}^{\mathbb{C}}(u)-E_{\mathbb{N} \mathbb{C}}^{\mathbb{C}}(u+t), \text { if }(n, t) \in u, t=0,1,2,3, \ldots, n .
$$

However, for $\delta E_{\mathbb{N} \mathbb{C}}^{\mathbb{C}}(u+u+t)=0$, there is no mobile sensor devices with in a cluster. $\delta E_{\mathbb{N} \mathbb{C}}^{\mathbb{C}}(u+u+$ $t)$ is a positive parameter for cluster member and $\mathrm{CH}$ moving towards each other; $\delta E_{\mathbb{N} \mathbb{C}}^{\mathbb{C}}(u+u+t)$ is a negative parameter for sensor devices in a cluster moving away from $\mathrm{CH}$. Then, the radio signal strength indicator parameter $J$ can be computed at any instace $u$ and $u+t$ as follows

$$
\begin{aligned}
& J_{\mathbb{N} \mathbb{C}}^{\mathbb{C}}(u)=J_{\mathbb{N} \mathbb{C}}^{c}(u)-J_{\mathbb{N} \mathbb{C}}^{\mathbb{M}} \\
& J_{\mathbb{N} \mathbb{C}}^{\mathbb{C}}(u+t)=J_{\mathbb{N} \mathbb{C}}^{c}(u+t)-J_{\mathbb{N} \mathbb{C}}^{\mathbb{M}}, \forall t \in u
\end{aligned}
$$

where $J_{\mathbb{N} C}^{c}$ is the present threshold parameter and $J_{\mathbb{N} C}^{\mathbb{M}}$ is the minimum threshold parameter at instance $u$ or $u+$ $t$. If $j$ is a positive parameter, the CMs associates with appropriate cluster and communicate with its respective $\mathrm{CH}$. Considering this scenario, the coverage time $\delta J_{\mathbb{N} \mathbb{C}}^{\mathbb{C}}(u+t)$ is the variance among $J_{\mathbb{N} \mathbb{C}}^{\mathbb{C}}(u)$ and $J_{\mathbb{N} \mathbb{C}}^{\mathbb{C}}(u+t)$, which is obtained using (6) as follows

$$
\delta J_{\mathbb{N} \mathbb{C}}^{\mathbb{C}}(u, u+r)=J_{\mathbb{N} \mathbb{C}}^{\mathbb{C}}(u)-J_{\mathbb{N} \mathbb{C}}^{\mathbb{C}}(u+t), \quad \forall t \in u,
$$

The cluster member is considered to be moving towards the $\mathrm{CH}$ whenever $\delta J_{\mathbb{N} \mathbb{C}}^{\mathbb{C}}(u, u+r) \geq 0$, and the $\mathrm{CM}$ is moving away from $\mathrm{CH}$ whenever $\delta J_{\mathbb{N} \mathbb{C}}^{\mathbb{C}}(u, u+r) \leq 0$. Post that, $H_{\mathbb{N} \mathbb{C}}^{n}$ is the value allocated to sensor device $\mathbb{N}$ for every round, which depicts the connectivity specifier with $\mathrm{CH} \mathbb{C}$. Considering this scenario, the $\mathbb{N}$ checks $H_{\mathbb{N C}}^{n}$ with one-hop neighbour node $\mathbb{C}$ to select ideal $\mathrm{CH}$ on each successive round. The dimensionless parameter $\Delta_{\mathbb{N} C}^{\mathbb{C}}, \alpha_{\mathbb{N} C}^{\mathbb{C}}, \beta_{\mathbb{N} \mathbb{C}}^{\mathbb{C}}$, and $L_{\mathbb{N} \mathbb{C}}^{\mathbb{C}}$ is a linear mixture with constant factor ranging between zero and one. The constant depicts the costs of each factor and are obtained as follows

$$
\Delta_{\mathbb{N} \mathbb{C}}^{\mathbb{C}}+\alpha_{\mathbb{N} \mathbb{C}}^{\mathbb{C}}+\beta_{\mathbb{N} \mathbb{C}}^{\mathbb{C}}+L_{\mathbb{N} \mathbb{C}}^{\mathbb{C}}=1
$$

Therefore, using (8) we can obtain $H_{\mathbb{N C}}^{n}$ as follows 


$$
H_{\mathbb{N} \mathbb{C}}^{n}=\left(\Delta_{\mathbb{N} \mathbb{C}}^{\mathbb{C}} * \frac{F_{O}^{\mathbb{M}}-F_{\mathbb{C}}^{c}}{F_{\mathbb{C}}^{C} * O_{\mathbb{C}}^{C}}\right)+\left(\alpha_{\mathbb{N} \mathbb{C}}^{\mathbb{C}} *\left(1-\frac{J_{\mathbb{N} \mathbb{C}}^{\mathbb{M}}}{J_{\mathbb{N} C}^{c}}\right)\right)+\left(\beta_{\mathbb{N} \mathbb{C}}^{\mathbb{C}} * \frac{e_{\mathbb{N}}-E_{\mathbb{N} \mathbb{C}}^{\mathbb{C}}(u)}{E_{\mathbb{N} \mathbb{C}}^{\mathbb{C}}(u)}\right)+\left(L_{\mathbb{N} \mathbb{C}}^{\mathbb{C}} * \frac{\delta u_{\mathbb{N} C}^{\mathbb{C}}}{u_{c}^{g}}\right),
$$

where $F_{\mathbb{C}}^{c}$ is the current energy level of $\mathrm{CH}, F_{O}^{\mathbb{M I}}$ is the initial energy, $O_{\mathbb{C}}^{c}$ is the number of present $\mathrm{CMs}$ for $\mathrm{CH}$ $\mathbb{C}, J_{\mathbb{N} C}^{c}$ is the present RSSI level ranging from $\mathbb{N}$ and $\mathbb{C}$ and, $J_{\mathbb{N C}}^{\mathbb{M}}$ is the minimum essential RSSI level from $\mathbb{N}$ and $\mathbb{C}, e_{\mathbb{C N}}$ is maximum coverage distance among $\mathbb{C}$ and $\mathbb{N}, E_{\mathbb{N} C}^{\mathbb{C}}(u)$ is the distance among $\mathbb{N}$ and $\mathbb{C}$ at any instance period $u, u_{c}^{g}$ is the present packet frame duration for $\mathbb{C}$ which is computed using (13) and $\delta u_{\mathbb{N} \mathbb{C}}^{\mathbb{C}}$ is the predicted connection time for $\mathbb{N}$ starts its transmission to $\mathbb{C}$.

\subsection{Packet/ traffic duration computation}

Here in this work, the number of expected CMs $N_{e}$ and number of present CMs $N_{c}$ on each round is obtained as follows

$$
\begin{aligned}
& N_{f}=\frac{O_{c}-D_{I}-D_{U}}{D_{I}}, \\
& O_{c}=O_{u}-O_{e}, \\
& N_{c}=N_{f}-\left(N_{o k}+N_{e}+N_{t}\right),
\end{aligned}
$$

where $N_{e}$ is the predictable number of $\mathrm{CM}, N_{c}$ is the current $\mathrm{CM}$ from one cluster, $O_{c}$ is the total number of current sensor device, $D_{I}$ is the $\mathrm{CH}, D_{U}$ is the total number of TPN, $O_{u}$ is the total number of sensor devices in WSN, $N_{t}$ is the total number of CM on sleep mode, $N_{f}$ is the number of sensor device dead, $N_{e}$ is the number of $\mathrm{CH}$ dead, and $N_{o k}$ is the newly joined CM from adjacent/neighbour cluster on this round. Therefore, the present time period of the data packet $u_{c}^{g}$ from each cluster is represented as follows

$$
u_{c}^{g}=\frac{M_{q}}{R_{b}} * N_{c} \text { or } u_{c}^{g}=\frac{M_{q}}{R_{b}} * N_{e}
$$

where $R_{b}$ is the transmission bit rate and $M_{q}$ is the data packet length.

\subsection{Transmission path formation communication}

The TPF communication stage starts post completion of intra cluster communication stage. In intra cluster stage, to form cluster, the sensor devices elects itself as $\mathrm{CH}$ to form a cluster, then the $\mathrm{CH}$ is fully responsible collecting data from cluster member, performing data aggregation and cluster maintenance. Post that, a transmission path formation is initialized, which connects $\mathrm{CH}$ to sink or base station. Now, the base station initialize the TPF creation process. Here based on connection time and location of $\mathrm{CH}$, a few sensor devices are chosen as transmission path node (TPN) to produce TPF. However, these sensor devices do no take part in sensing and does not belongs to any cluster on that specific round. As a result, may act as a normal sensor device. Considering this scenario, the selection of TPN does not affects data communication of respective cluster. It should have good connection time among nearest TPN and CH. The TPF creation is based on connection, coverage time, location of $\mathrm{CH}$, and connectivity (i.e., among the $\mathrm{CH}$ and TPN). It clear in TPN communication. After the initialization stage (i.e., ahead intra cluster and TPF), packet transmission is commenced in the transmission stage. Here, all the CMs transmit the data towards base station on the ideal path.

\subsection{Transmission path formation creation}

TPF is a hierarchical path structure, which utilize TPN to collect the packet among CHs and transmit to base station, and that covers the entire wireless sensor networks. Here, the base station chooses the TPN based on the threshold parameter, radio signal strength, connection time, communication range, and connectivity which aid in reducing the energy overhead and traffic overhead of the entire wireless sensor network. If the sensor device are of high mobile in nature, then the selected TPF can keep the association with the $\mathrm{CH}$ for a longer instance and there is no necessity to update in the path structure. For improving the lifetime of network a new TPN is selected every instance a new $\mathrm{CH}$ are elected (i.e., the new $\mathrm{CH}$ and TPN is chosen on every round). The selection of New TPN is performed by base station, which is based on the mobility of the new $\mathrm{CH}$. The TPN collects the packet form $\mathrm{CH}$, aggregates the data by removing duplicate information and then transmit the data packet to the next or adjacent TPN. 


\subsection{Transmission path node communication}

Post completion of intra cluster communication stage, TPN communication is initialized. Here, base station chooses the first TPN based on threshold parameter, coverage time, connection time, connectivity, and network traffic/packet duration TPF of the TPN. Post that, TPN checks the CH in one-hop distance and creates next TPN to extent the routing/transmission path/tree structure. Considering the scenario if no $\mathrm{CH}$ is available in one-hop distance, then it will just elect alternative TPN in one-hop distance to search the $\mathrm{CH}$ and extend the routing path structure. Subsequently, TPF makes a communication link among $\mathrm{CH}$ and base station. To identify the TPN on TPF, the threshold value is computed by adding the count of preceding neighbour device or TPN from the base station with multiplied factor such as remaining energy, current coverage and speed of sensor device (i.e., $\mu$ is considered to identify the TPN from the next hop or neighbour device) as follows

$$
U_{\gamma \mu}^{q}=I_{\gamma}+\left(\frac{F_{O}^{\mathbb{M}}-F_{\mu}^{c}}{F_{O}^{\mathbb{M}}} * \frac{W_{O}^{\mathbb{M}}-W_{\mu}^{c}}{V_{O}^{\mathbb{M}}+W_{\mu}^{c}} * \frac{S_{O}^{\mathbb{M}}-S_{\mu}^{c}}{S_{O}^{\mathbb{M}}+S_{\mu}^{c}}\right)
$$

where $I_{\gamma}$ is the hop count of $\gamma$ from base station in TPF (i.e., $\gamma$ is supposed to identify the preceding TPF or neighbour device in TPF), $F_{O}^{\mathbb{M}}$ is the initial energy of sensor device, $F_{\mu}^{c}$ is the current energy of $\mu, W_{O}^{\mathbb{M}}$ is the maximum speed of sensor device, $W_{\mu}^{c}$ is the current speed of $\mu, S_{O}^{\mathbb{M}}$ is the maximum coverage radius of sensor device, and $S_{\mu}^{c}$ is the current coverage radius of $\mu$.

\subsection{Transmission Stage}

Post completion of initialization stage, transmission stage is initialized. In transmission stage, all the $\mathrm{CMs}$ transmit the gathered data its respective $\mathrm{CH}$ in its respective slotted time. Then, the $\mathrm{CH}$ starts gathering data and aggregates the data from its CMs and in mean time TPF communication is initialized, which utilized spectrum [32] to transfer the packet from $\mathrm{CH}$ to TPN and then towards base stations. Here, the TPN gathers and aggregates the data from the respective $\mathrm{CH}$ or TPN.

\section{SIMULATION RESULT AND ANNALYSIS}

This section present performance of proposed LLEER model over existing algorithm under various parameter through simulation. The system environment used for experiment analysis is windows 10 enterprises edition operating system, Intel Pentium I-5 class, 64-bit Quad core processor, 4GB NVIDIA CUDA enabled dedicated graphic card, 16GB RAM. The SENSORIA simulator [33] is used to carry out performance evaluation of LLEER over existing approaches such as LEACH [11]. The SENSORIA Simulator and LLEER and LEACH is designed using C\# programming language. This work consider LEACH protocol as a case study for comparison. Since, it is been used a standard for comparison by many exiting approaches [11]. The wireless sensor network is composed of 400 to 1200 sensor devices. All the sensor device are randomly placed across square region of $50 * 50$ square meters which is static in nature, the base station is placed at edge of the network (i.e., $\mathrm{x}=0, \mathrm{y}=0$ ) outside sensing area. The size of data packet is set to 2000 bits, transmission speed is set to $100 \mathrm{bit} / \mathrm{second}$ (s), bandwidth is set to $5000 \mathrm{bit} / \mathrm{second}$, sensing range is set to maximum of 3 meter (m), transmission range is set to maximum of $5 \mathrm{~m}$ with in cluster, and data packet processing delay is set to 0.1 seconds. Further communication energy parameter such as idle energy consumption (Eelec) is set to $50 \mathrm{nj} / \mathrm{bit}$, Radio energy dissipation is set to $50 \mathrm{nj} / \mathrm{bit}$, Amplification energy (Emp) is set to $100 \mathrm{pJ} / \mathrm{bit} / \mathrm{m} 2$, and Initial energy of sensor devices is set to $0.1 \mathrm{~J}$ to $0.2 \mathrm{~J}$.

\subsection{Lifetime performance evaluation for total sensor device death}

Here we obtained network lifetime performance of LLEER and LEACH considering total sensor device death under varied sensor devices which is shown in Figure 2. From figure it can be seen that LLEER achieves significant lifetime performance over LEACH considering varied sensor device. A lifetime improvement of $77.68 \%, 75.4 \%, 80.82 \%, 83.57 \%$, and $82.41 \%$ is achieved by LLEER over LEACH protocol considering 400, 600, 800, 1000, and 1200 sensor devices respectively. An average lifetime improvement of $79.78 \%$ is achieved by LLEER over LEACH protocol. From experiment outcome it can be seen as sensor device increases the lifetime performance of LEACH degrades and LLEER stable which shows LEACH is not efficient for high density network and LLEER is adaptive in nature irrespective of sensor device size. 


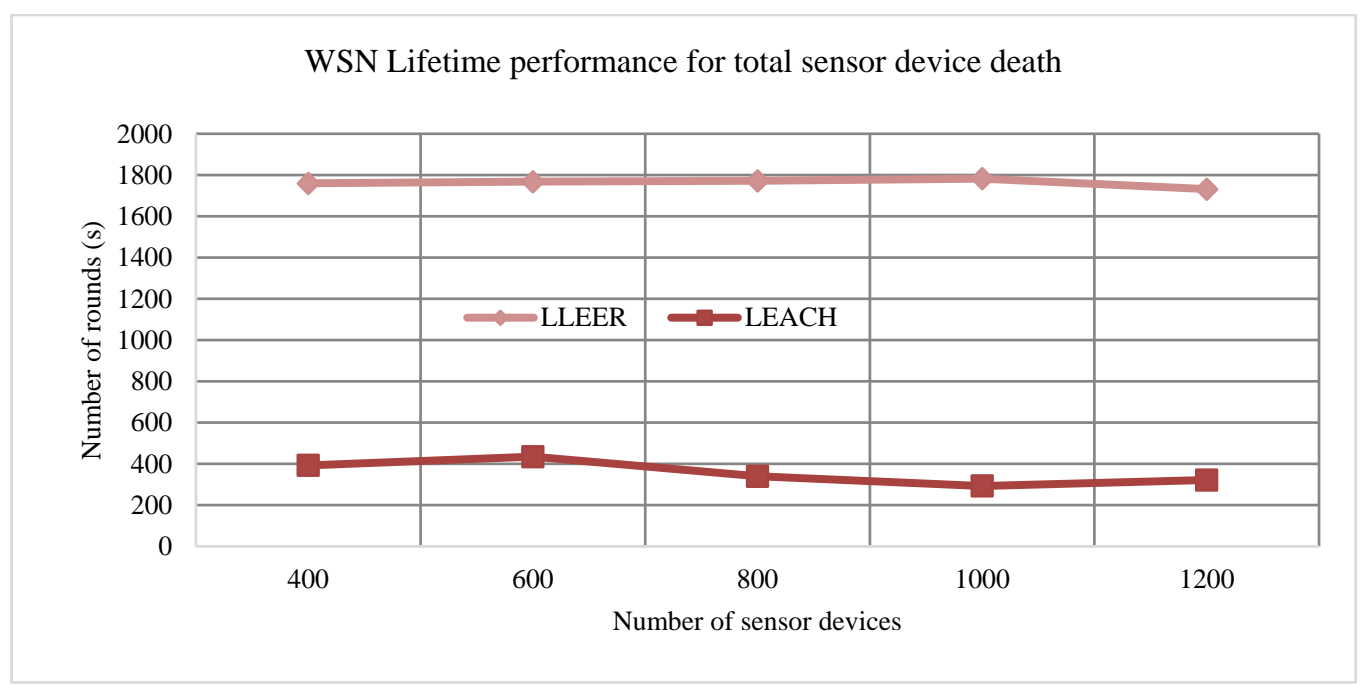

Figure 2. WSN lifetime performance for total sensor device death

\subsection{Lifetime performance evaluation for first sensor device death}

Here we obtained network lifetime performance of LLEER and LEACH considering first sensor device death under varied sensor devices which is shown in Figure 3. From figure it can be seen that LLEER achieves significant lifetime performance over LEACH considering varied sensor device. A lifetime improvement of $81.86 \%, 77.51 \%, 87.89 \%, 91.76 \%$, and $89.17 \%$ is achieved by LLEER over LEACH protocol considering 400, 600, 800, 1000, and 1200 sensor devices respectively. An average lifetime improvement of $85.64 \%$ is achieved by LLEER over LEACH protocol. From experiment outcome it can be seen as sensor device increases the lifetime performance of LEACH degrades and LLEER stable which shows LEACH is not efficient for high density network and LLEER is adaptive in nature irrespective of sensor device size.

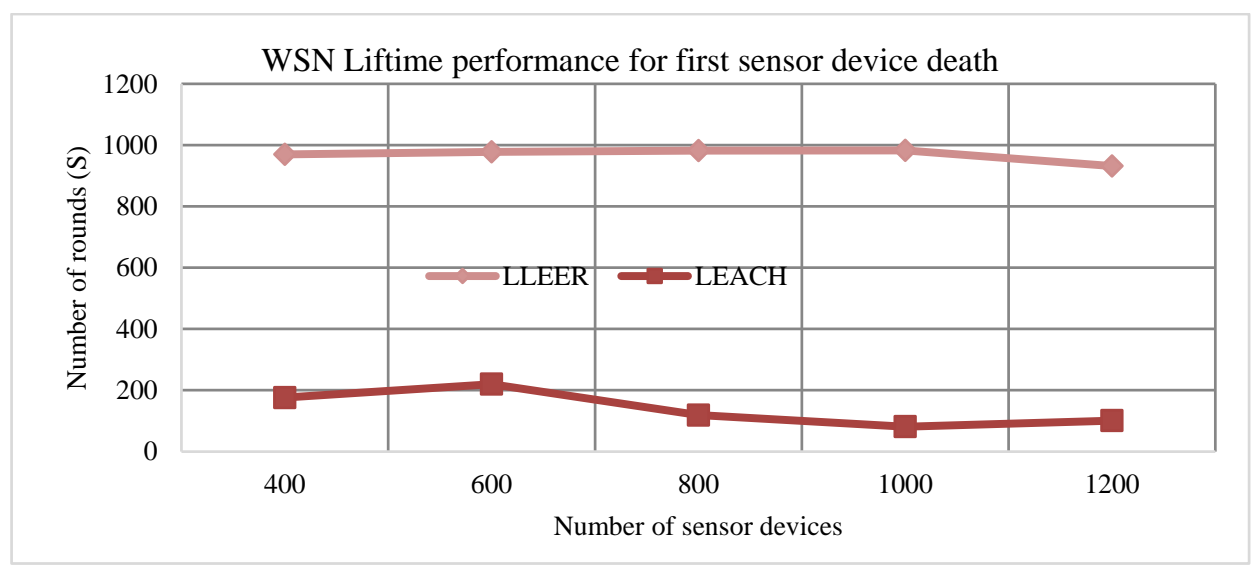

Figure 3. WSN lifetime performance for first sensor device death

\subsection{Lifetime performance evaluation for loss of connectivity}

Here we obtained network lifetime performance of LLEER and LEACH considering loss of connectivity under varied sensor devices which is shown in Figure 4. From figure it can be seen that LLEER achieves significant lifetime performance over LEACH considering varied sensor device. A lifetime improvement of $81.57 \%, 79.42 \%, 85.63 \%, 87.23 \%$, and $86.07 \%$ is achieved by LLEER over LEACH protocol considering 400, 600, 800, 1000, and 1200 sensor devices respectively. An average lifetime improvement of $85.78 \%$ is achieved by LLEER over LEACH protocol. From experiment outcome it can be seen as sensor device increases the lifetime performance of LEACH degrades and LLEER stable which shows LEACH is not efficient for high density network and LLEER is adaptive in nature irrespective of sensor device size.

Low latency and energy efficient cluster based routing design for wireless sensor network (Basavaraj G.N) 


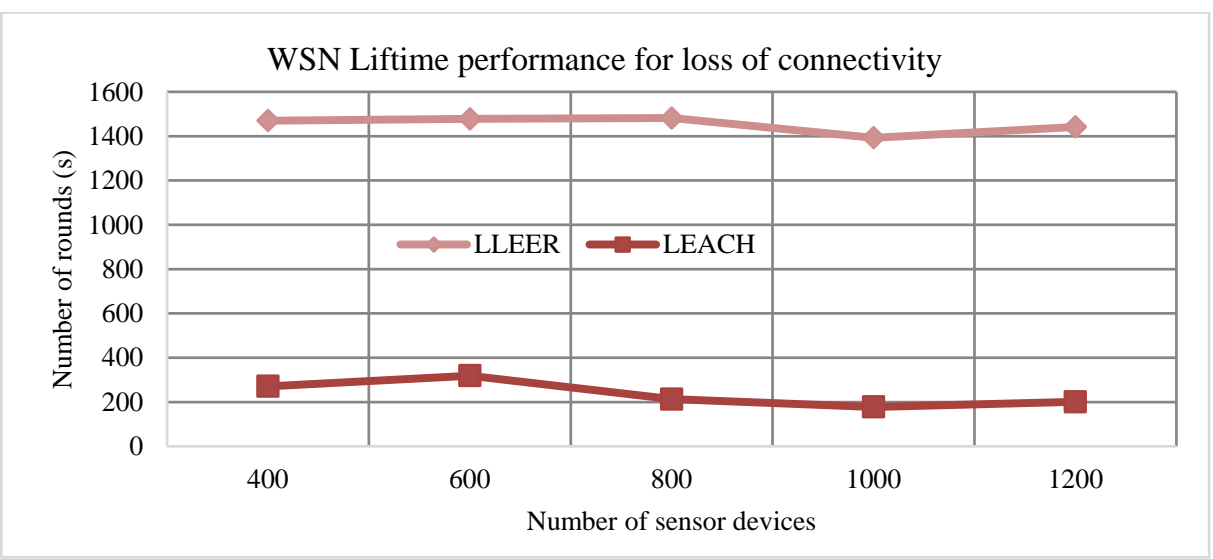

Figure 4. WSN lifetime performance for first sensor device death

\subsection{Communication overhead performance}

Here we obtained communication overhead performance of LLEER and LEACH considering total sensor device death under varied sensor devices which is shown in Figure 5. From figure it can be seen that LLEER achieves significant reduction in communication overhead over LEACH considering varied sensor device. An communication overhead reduction of $24.92 \%, 19.13 \%, 48.87 \%, 41.88 \%$, and $47.39 \%$ is achieved by LLEER over LEACH protocol considering 400, 600, 800, 1000, and 1200 sensor devices respectively. An average communication overhead reduction of $36.19 \%$ is achieved by LLEER over LEACH protocol. From experiment outcome it can be seen as sensor device increases the communication overhead performance of LEACH degrades significantly when compared to LLEER which shows LEACH is not efficient for high density network and LLEER is adaptive in nature irrespective of sensor device size.

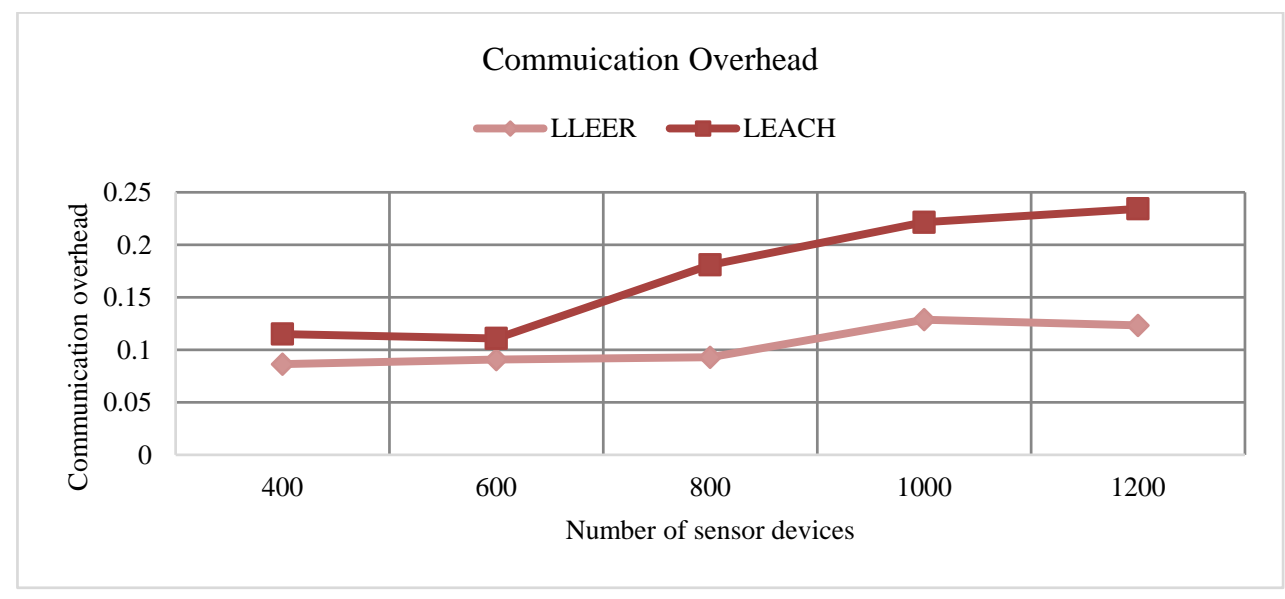

Figure 5. Communication overhead performance considering varied sensor device

\subsection{Packet transmission latency performance}

Here we obtained packet transmission latency performance of LLEER and LEACH considering total sensor device death under varied sensor devices which is shown in Figure 6. From figure it can be seen that LLEER achieves significant reduction in transmission latency over LEACH considering varied sensor device. A transmission latency reduction of $42.24 \%, 48.64 \%, 48.95 \%, 41.54 \%$, and $47.72 \%$ is achieved by LLEER over LEACH protocol considering 400, 600, 800, 1000, and 1200 sensor devices respectively. An average transmission latency reduction of $45.42 \%$ is achieved by LLEER over LEACH protocol. From experiment outcome it can be seen as sensor device increases the transmission latency performance of LEACH degrades significantly when compared to LLEER which shows LEACH is not efficient for high density network and LLEER is adaptive in nature irrespective of sensor device size. 


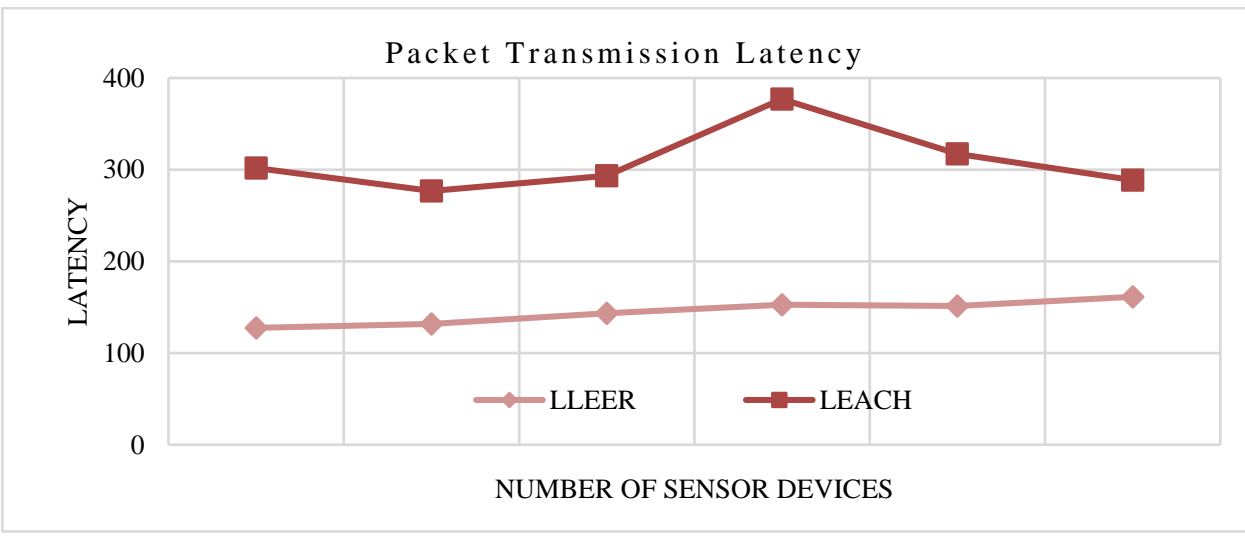

Figure 6. Packet transmission latency performance considering varied sensor device

\section{RESULT AND DISCUSSION}

This work carried out experiment analysis on various performance parameter such as network lifetime, communication overhead and latency incurred and compared result over existing approaches. Most state-of-art technique considered total sensor device death as performance parameter to evaluate their model. However, performance evaluation considering first sensor device death is also very vital parameter as it leads to loss of connectivity among sensor devices and affecting lifetime performance of wireless sensor network. As a result, this paper considers lifetime performance evaluation considering total sensor device death, first sensor device death and loss of connectivity. Below in Table 1 performance comparison of proposed LLEER and existing protocols lifetime achievement over LEACH protocol is tabulated. The overall outcome shows the LLEER model achieved significant network lifetime performance improvement over state-of-art model [18], [19], [29], [31], and [34] considering total node death, first node death and loss of connectivity. Significant outcome achieved is due to adoption of cluster and hop based transmission using multi objective parameter. Our model brings a good trade-off between energy efficiency and latency minimization. Thus will aid in provision realtime data access application services.

Table 1. Perrformance Comparison Of Network Lifetime Achievement Over LEACH

\begin{tabular}{cccc}
\hline Algorithm & $\begin{array}{c}\text { Lifetime improvement achieved } \\
\text { over LEACH considering total } \\
\text { node death }\end{array}$ & $\begin{array}{c}\text { Lifetime improvement achieved over } \\
\text { LEACH considering first node death }\end{array}$ & $\begin{array}{c}\text { Lifetime improvement achieved over } \\
\text { LEACH considering loss of connectivity }\end{array}$ \\
\hline$[18]$ & $25.0 \%$ & $56.7 \%$ & - \\
{$[19]$} & $50.0 \%$ & - & - \\
{$[29]$} & $55.0 \%$ & - & - \\
{$[31]$} & $44.0 \%$ & - & - \\
{$[34]$} & $15.0 \%$ & $85.64 \%$ & $85.84 \%$ \\
LLEER & $79.78 \%$ & & \\
\hline
\end{tabular}

\section{CONCLUSION}

Building low latency and energy efficient WSN is most desired. Clustering technique play a significant part in minimizing energy consumption sensor device and enhancing lifetime of senor network. Recently, number of hierarchical clustering approach has been presented to minimize energy by enhancing LEACH. The drawback of state-of-art design is it incurs energy overhead among cluster head due to long transmission range, degrades energy of sensor device closer to base station and are presented considering homogenous network. However, the future application such as big data and IoT requires low latency real-time data access. These application are designed considering heterogeneous WSN environment and very limited work is carried out considering heterogeneous network. This work presented LLEER which minimize energy consumption, maximize lifetime and reduce data transmission latency. The LLEER adopts multi-objective function such as connectivity, connection time, radio signal strength, coverage time, and network traffic for cluster head and hop node selection. Experiment are conducted to evaluate the performance of LLEER over LEACH protocol. Existing work predominantly consider performance evaluation considering total node death and very limited work is carried out to evaluate performance in terms of first node death and loss of connectivity. The experiment outcome shows an average network lifetime performance improvement of $79.78 \% 85.64 \%$, and $85.84 \%$ is attained by LLEER over LEACH protocol considering total node death, first

Low latency and energy efficient cluster based routing design for wireless sensor network (Basavaraj G.N) 
node death, and loss of connectivity respectively. Further, communication overhead and packet transmission latency performance is evaluated. The outcome shows LLEER reduces communication overhead and packet transmission latency by $36.19 \%$ and $45.42 \%$ over LEACH protocol. This work also presented cumulative analysis to evaluate lifetime performance of LLEER over state-of-art model (such as Nayak et al., 2016; Nayak et al., 2017; Twayej et al., 2017; Hong et al., 2016; \& Deva, Mall, \& Kar 2016). The outcome shows significant performance improvement of LLEER over state-of-art model in terms of network lifetime considering total node death, first node death, and loss of connectivity, communication overhead, and packet transmission latency. Proposed LLEER brings a good trade-off between energy efficiency, and latency requirement of future real-time application.

\section{REFERENCES}

[1] M. R. Palattella et al., "Internet of Things in the 5G era: Enablers, architecture, and business models," IEEE J. Sel. Areas Commun., vol. 34, no. 3, pp. 510-527, Mar. 2016.

[2] N. M. Abdul Latiff*, M. A. Ruslee, S. K. Syed Yusof, M. R. Abdul Rahim, H. Sayuti, K. Mohamad Yusof, M. A. Baharudin, Indonesian Journal of Electrical Engineering and Computer Science (IJEECS) Vol. 6, No. 1, April 2017 pp. $80 \sim 87$.

[3] A. Zanella, N. Bui, A. Castellani, L. Vangelista, and M. Zorzi, "Internet of Things for smart cities," IEEE Internet Things J., vol. 1, no. 1, pp. 22-32, Feb. 2014.

[4] Wu Chenjian, Li Zhiqun, Yao Nan, Zhang Meng, Chen Liang, Cao Jia Institute of of RF- \& OE-ICs, Southeast University, Nanjing, 210096. TELKOMNIKA (Telecommunication Computing Electronics and Control), Vol. 11, No. 8 TELKOMNIKA (Telecommunication Computing Electronics and Control), Vol. 11, No. 8, August 2013, pp. 4470 4476.

[5] J. Fan, F. Han, and H. Liu, "Challenges of big data analysis," Nat. Sci. Rev., vol. 1, no. 2, pp. 293-314, 2014.

[6] M. Peng, S. Yan, K. Zhang, and C. Wang, "Fog-computing-based radio access networks: Issues and challenges," IEEE Netw., vol. 30, no. 4, pp. 46-53, Jul./Aug. 2016.

[7] Y. Shi, J. Zhang, and K. B. Letaief, "Group sparse beamforming for green cloud-RAN," IEEE Trans. Wireless Commun., vol. 13, no. 5, pp. 2809-2823, May 2014.

[8] Y. Shi, J. Zhang, K. B. Letaief, and W. Chen, "Large-scale convex optimization for ultra-dense cloud-RAN," IEEE Wireless Commun., vol. 22, no. 3, pp. 84-91, Jun. 2015.

[9] M. Chiang and T. Zhang, "Fog and IoT: An overview of research opportunities," IEEE Internet Things J., vol. 3, no. 6, pp. 854-864, Dec. 2016.

[10] G. Anastasi, M. Conti, M. Di Francesco, and A. Passarella, "Energy conservation in wireless sensor networks: A survey," Ad Hoc Netw., vol. 7, no. 3, pp. 537-568, May 2009.

[11] I. F. Akyildiz, W. Su, Y. Sankarasubramaniam, and E. Cayirci, "Wireless sensor networks: A survey," Comput. Netw., vol. 38, no. 4, pp. 393-422, Mar. 2002.

[12] A. Nayak and I. Stojmenovic, Wireless Sensor and Actuator Networks: Algorithms and Protocols for Scalable Coordination and Data Communication. Hoboken, NJ, USA: Wiley, 2010.

[13] W. Dargie, "Dynamic power management in wireless sensor networks: State-of-the-art," IEEE Sensors J., vol. 12, no. 5, pp. 1518-1528, May 2012.

[14] M. Saleem, G. A. Di Caro, and M. Farooq, "Swarm intelligence based routing protocol for wireless sensor networks: Survey and future directions," Inf. Sci., vol. 181, no. 20, pp. 4597-4624, Oct. 2011

[15] J. Yick, B. Mukherjee, and D. Ghosal, "Wireless sensor network survey," Comput. Netw., vol. 52, no. 12, pp. 2292 2330, Aug. 2008.

[16] D. V. Puspalata and P. Nayak, "A clustering algorithm for WSN to optimize the network lifetime using type-2 fuzzy logic model," in Proc. 3rd Int. Conf. Artif. Intell., Modeling Simulations (AIMS), Kota Kinabalu, Malaysia, Dec. 2015, pp. 53-58.

[17] S. Bandyopadhyay and E. J. Coyle, "An energy efficient hierarchical clustering algorithm for wireless sensor networks," in IEEE INFOCOM, vol. 3, 2003.

[18] P. Nayak and A. Devulapalli, "A Fuzzy Logic-Based Clustering Algorithm for WSN to Extend the Network Lifetime," in IEEE Sensors Journal, vol. 16, no. 1, pp. 137-144, Jan.1, 2016.

[19] P. Nayak and B. Vathasavai, "Energy Efficient Clustering Algorithm for Multi-Hop Wireless Sensor Network Using Type-2 Fuzzy Logic," in IEEE Sensors Journal, vol. 17, no. 14, pp. 4492-4499, July15, 152017.

[20] U. Srinivasan and B. Arunasalam, "Leveraging big data analytics to reduce healthcare costs," IT Prof., vol. 15, no. 6, pp. 21-28, Nov./Dec. 2013.

[21] N. Sun, J. G. Morris, J. Xu, X. Zhu, and M. Xie, "iCARE: A framework for big data-based banking customer analytics," IBM J. Res. Develop., vol. 58, nos. 5/6, pp. 4:1-4:9, 2014.

[22] C. Sun, R. Gao, and H. Xi, "Big data based retail recommender system ofnon E-commerce," in Proc. Int. Conf. Comput., Commun. Netw. Technol.,2014, pp. 1-7.

[23] [Online]. Available: http://www.ibm.com/big-data/au/en/big-data-andanalytics/ operations-management.html

[24] K. L. M. Ang; J. K. P. Seng; A. M. Zungeru, "Optimizing Energy Consumption for Big Data Collection in LargeScale Wireless Sensor Networks With Mobile Collectors," in IEEE Systems Journal , vol.PP, no.99, pp.1-11. 
[25] S. Rani, S. H. Ahmed, R. Talwar and J. Malhotra, "Can Sensors Collect Big Data? An Energy-Efficient Big Data Gathering Algorithm for a WSN," in IEEE Transactions on Industrial Informatics, vol. 13, no. 4, pp. 1961-1968, Aug. 2017.

[26] X. Liu, J. Li, Z. Dong and F. Xiong, "Joint Design of Energy-Efficient Clustering and Data Recovery for Wireless Sensor Networks," in IEEE Access, vol. 5, no. , pp. 3646-3656, 2017.

[27] Chandramouli H, Dr. Somashekhar C Desai, K S Jagadeesh, Kashyap D Dhruve. "Enhancing Network Lifetime in Wireless Sensor Networks Adopting Elephant Swarm Optimization", Global Journal of Computer Science and Technology, NETWORK, WEB \& SECURITY, Volume 13, Issue 4, Version 1.0, pp 09-17, Mar 2013.

[28] Chandramouli H, Dr. Somashekhar C Desai, K S Jagadeesh, Kashyap D Dhruve. "Elephant Swarm Optimization for Wireless Sensor Networks - A Cross Layer Mechanism”, International Journal of Computer Science \& Technology (IJCET), Volume 4, Issue 2, pp 45-60, March - April 2013.

[29] W. Twayej, M. Khan and H. S. Al-Raweshidy, "Network Performance Evaluation of M2M With Self Organizing Cluster Head to Sink Mapping," in IEEE Sensors Journal, vol. 17, no. 15, pp. 4962-4974, Aug.1, 12017.

[30] C. W. Tsai, T. P. Hong and G. N. Shiu, "Metaheuristics for the Lifetime of WSN: A Review," in IEEE Sensors Journal, vol. 16, no. 9, pp. 2812-2831, May1, 2016.

[31] Z. Hong, R. Wang and X. Li, "A clustering-tree topology control based on the energy forecast for heterogeneous wireless sensor networks," in IEEE/CAA Journal of Automatica Sinica, vol. 3, no. 1, pp. 68-77, January 102016.

[32] Ghaisari, Jafar \& Ferdosi, Arash. (2011). A direct sequence spread spectrum code acquisition circuit for wireless sensor networks. International Journal of Electronics. 98. 793-800. 10.1080/00207217.2010.547813.

[33] J. N. Al-Karaki and G. A. Al-Mashaqbeh, "SENSORIA: A New Simulation Platform for Wireless Sensor Networks," 2007 International Conference on Sensor Technologies and Applications (SENSORCOMM 2007), Valencia, 2007, pp. 424-429.

[34] H. K. Deva Sarma, R. Mall and A. Kar, "E2R2: Energy-Efficient and Reliable Routing for Mobile Wireless Sensor Networks," in IEEE Systems Journal, vol. 10, no. 2, pp. 604-616, June 2016. 